\title{
Delayed initiation of radiation therapy is associated with inferior outcomes for breast cancer patients with hormone receptor- negative tumors after breast-conserving surgery
}

\author{
Xiaoyan $\mathrm{Ma}^{1,2 \#}$, Jie Chen ${ }^{3 \#}$, Ding $\mathrm{Ma}^{1,2 \#}$, Yizhou Jiang ${ }^{1,2}$, Zhebin Liu ${ }^{1,2}$, Sheng Chen ${ }^{1,2}$, Ying Zhang ${ }^{1,2}$, \\ Yizhou Shen ${ }^{1,2}$, Xiaoli Yu ${ }^{2,4}$, Zhaozhi Yang, ${ }^{2,4}$, Shuang $\mathrm{Li}^{5}$, Miao Mo ${ }^{2,6}$, Yongxiang Qian ${ }^{3}$, Guangyu Liu ${ }^{1,2}$, \\ Jiong $\mathrm{Wu}^{1,2}$, Zhiming Shao ${ }^{1,2}$, Keda Yu ${ }^{1,2}$, Genhong $\mathrm{Di}^{1,2}$ \\ ${ }^{1}$ Department of Breast Surgery, Key Laboratory of Breast Cancer in Shanghai, Fudan University Shanghai Cancer Center, Shanghai, China; \\ ${ }^{2}$ Department of Oncology, Shanghai Medical College, Fudan University, Shanghai, China; ${ }^{3}$ Department of Thyroid and Breast Surgery, First \\ People's Hospital of Taicang, Taicang, China; ${ }^{4}$ Department of Radiology, Fudan University Shanghai Cancer Center, Shanghai, China; ${ }^{5}$ Department \\ of Breast Surgery, Liaoning Province Cancer Hospital, Shenyang, China; ${ }^{6}$ Department of Cancer Prevention, Fudan University Shanghai Cancer \\ Center, Shanghai, China \\ Contributions: (I) Conception and design: G Di, K Yu, Z Liu; (II) Administrative support: Z Shao, J Wu, G Liu, X Yu; (III) Provision of study \\ materials or patients: G Di, K Yu, Z Liu, X Ma, J Chen, D Ma; (IV) Collection and assembly of data: X Ma, J Chen, D Ma, S Chen, Y Zhang, Y \\ Shen, Z Yang, S Li, Y Qian; (V) Data analysis and interpretation: X Ma, J Chen, D Ma, Y Jiang, M Mo; (VI) Manuscript writing: All authors; (VII) \\ Final approval of manuscript: All authors. \\ "These authors contributed equally to this work. \\ Correspondence to: Dr. Genhong Di; Dr. Keda Yu; Dr. Zhebin Liu. Department of Breast Surgery, Cancer Center, Shanghai Medical College, Fudan \\ University, 270 Dong An Road, Shanghai 200032, China. Email: dgh_2015@126.com; yukeda@163.com; lysalzb@hotmail.com.
}

Background: To investigate whether the interval between adjuvant chemotherapy (CT) completion and postoperative radiation therapy initiation (ICR) after breast-conserving surgery (BCS) affects ipsilateral breast tumor recurrence (IBTR) or survival.

Methods: All women who were diagnosed with invasive breast cancer and underwent BCS between 2005 and 2014 were included. In total, 1,472 patients underwent adjuvant CT followed by postoperative radiation therapy (RT) (CT+), whereas 402 patients received postoperative RT alone (CT-). Analyses were stratified by ICR and the interval between surgery and the initiation of postoperative RT (ISR) in these two cohorts. The cutoff points for treatment delay were 47 days in the CT+ cohort and 69 days in the CT- cohort. IBTR, local-regional failure (LRF), disease-free survival (DFS), and overall survival (OS) were assessed through Kaplan-Meier (K-M) analysis. Univariate and multivariate regression analyses were performed to determine the prognostic factors of survival outcomes.

Results: The median follow-up duration was 56 months. There was an association between a delay in ICR and an increase in IBTR in the CT+ group ( $\mathrm{P}=0.014$ for intervals $\leq 47 v s$. $>47$ days). This association was confirmed by multivariate analyses [hazard ratio $(\mathrm{HR})$ of $2.766 ; \mathrm{P}=0.046$ ] in the hormone receptornegative subgroup. The 5-year cumulative incidence rates of IBTR were $1.3 \%$ and $3.3 \%$ ( $\leq 47$ vs. $>47$ days, respectively) in the CT+ cohort. For patients in the CT- cohort, a longer delay of initiation of postoperative RT ( $\leq 69 v s .>69$ days) significantly decreased DFS (HR of $6.430 ; \mathrm{P}=0.002$ ). The 5 -year cumulative incidence rates of disease recurrence were $3.0 \%$ for RT starting $\leq 69$ days after surgery and $12.6 \%$ for RT starting $>69$ days after surgery.

Conclusions: A high IBTR rate was related to an ICR beyond 47 days. Delay of RT after CT or surgery among patients who undergo BCS should be avoided, especially among patients in the hormone receptornegative subgroup.

Keywords: Breast-conserving surgery (BCS); local recurrence; radiation therapy (RT); surgery-radiotherapy interval; delay 
Submitted Sep 14, 2020. Accepted for publication Jul 30, 2021.

doi: $10.21037 /$ gs-20-717

View this article at: https://dx.doi.org/10.21037/gs-20-717

\section{Introduction}

Radiation therapy (RT) is a necessary treatment for patients who undergo breast-conserving surgery (BCS) since BCS plus radiation achieves the same survival outcomes as mastectomy (1-4). It remains unknown whether RT should start within a specific interval after BCS to achieve optimal local control. Knauerhase et al. reviewed the interval between surgery and RT ( $\leq 2 v s .>2$ months) among 263 women who treated with BCS and RT for invasive breast cancer. The study found that the interval was a significant risk factor for ipsilateral breast tumor recurrence (IBTR) among the patients (5). Shurell et al. suggested that women who undergo BCS for ductal carcinoma in situ (DCIS) should start RT within 12 weeks. Their study showed that delayed initiation of RT was associated with a significantly higher risk of IBTR than early initiation of RT (6). A meta-analysis illustrated that patients who underwent BCS and delayed initiation of RT for more than 24 weeks had increased rates of local recurrence and distant metastases when adjuvant chemotherapy (CT) was administered first (7). Additionally, another study analyzed 18,050 women aged over 65 years who underwent BCS and RT and found that initiation of RT over 6 weeks after surgery was associated with an increased risk of local recurrence (8).

However, some previous studies reported that delayed initiation of RT did not lead to an increased risk of IBTR. A study of a low-risk group of women who did not receive adjuvant CT found no significant difference in IBTR within intervals of 0 to 20 weeks from BCS to the start of RT. The author reported the imbalance of factors related to local recurrence and thus suggested that patients had differential access to RT, which might explain the negative results between IBTR and delayed RT (9). Another study reported that the delayed interval of RT initiation was not significantly different in either early breast cancer or locally advanced breast cancer $(10,11)$. In addition, two studies defined cutoff points of delayed initiation of RT as 6 and 7 months and found that delayed onset of RT was not significantly associated with IBTR compared to patients who initiated RT earlier $(12,13)$. These studies speculated that confounding factors, such as age at diagnosis (10), wound healing or seroma formation (12), could lead to delayed onset of RT, which might contribute to negative results.

For ethical reasons, a randomized controlled trial cannot be conducted to assess the effect of the initiation timing of RT on survival outcomes. Some of the studies mentioned above were retrospective, mono-institutional studies, or meta-analyses, leading to the inconsistent definition of delayed RT. To address the inconsistency, a larger evaluation of this issue is required.

The relevance of the optimal timing of initiation RT and survival is widely described in the above-mentioned literature. Even if the results are statistically significant, further clinical trials and longer follow-up periods are warranted to validate their clinical significance. In this study, we retrospectively analyzed 1874 women who were treated with postoperative RT after BCS. The objective of the current study was to assess the association between the timing of postoperative RT and the risk of IBTR in women undergoing BCS.

We present the following article in accordance with the STROBE reporting checklist (available at https://dx.doi. org/10.21037/gs-20-717).

\section{Methods}

\section{Participants}

Women who were diagnosed with stage I-IIIc invasive breast cancer and received BCS at the Fudan University Shanghai Cancer Center (FUSCC) from February 2005 to December 2014 were included in this study. The study was conducted in accordance with the Declaration of Helsinki (as revised in 2013). All patients included in this study were inpatients, and these programs were approved by the Ethics Committee Review Board of Fudan University Shanghai Cancer Center (No. IRB050432-4-1911D), and each participant signed an informed consent form. Patients for whom basic clinical characteristics were unavailable, such as hormone receptor status, tumor size, margin, and lymph node status, were excluded.

Overall, 2,236 patients met the initial eligibility criteria. Three hundred and eleven patients who met all 
of the selection criteria but did not receive postoperative RT were excluded. Forty patients with ambiguous dates regarding the completion of adjuvant $\mathrm{CT}$ and the initiation of postoperative RT were also excluded. Patients who experienced a prolonged interval between surgery and the first dose of adjuvant CT ( $>8$ weeks) were also excluded $(\mathrm{n}=11)$. The final cohort included 1,874 patients. The patients were divided into a CT+ cohort $(\mathrm{n}=1,472)$ and a CT - cohort ( $\mathrm{n}=402$ ) according to whether they received adjuvant CT prior to postoperative RT (Figure S1).

Time to RT was defined as follows. In patients who received adjuvant $\mathrm{CT}$ and postoperative $\mathrm{RT}$ after BCS, the interval was between the completion of adjuvant CT and the initiation of postoperative radiation therapy (ICR). In patients who received postoperative RT without adjuvant CT after BCS, the interval was between surgery and the initiation of postoperative RT (ISR).

\section{Management}

All patients received whole breast irradiation by $3 \mathrm{D}$ conformal radiotherapy (3D-CRT) or intensity-modulated radiotherapy (IMRT) and a total dose of 50 Gy in 25 daily fractions. The postoperative tumor bed was delineated based on the clips and seroma combined with other postoperative changes. The boost clinical target volume was defined as uniformly adding a margin of $10 \mathrm{~mm}$ around the tumor bed. The boost dose for the tumor bed was 10-16 Gy, by $2 \mathrm{~Gy} /$ fraction. Post-surgery lymphatic RT was recommended to patients with four or more positive nodes and/or patients with one to three positive nodes associated with certain high-risk pathologic features.

Pathologic examination of tumor specimens was carried out in the Department of Pathology at FUSCC. Estrogen receptor (ER) and progesterone receptor (PR) assays were considered positive if they had an expression rate equal to or more than $10 \%$ for a specimen (14). ER- and PRwere defined as hormone receptor-negative, while ER+/ $\mathrm{PR}+, \mathrm{ER}+/ \mathrm{PR}-$, and $\mathrm{ER}-/ \mathrm{PR}+$ were defined as hormone receptor-positive. Human epidermal growth factor receptor 2 (HER2) status was recognized as positive when immunohistochemistry (IHC) staining of the $3+$ or HER2 gene was amplified by fluorescent in situ hybridization (FISH) analysis (15). Adjuvant systematic treatment of breast cancer (including a variety of chemotherapeutic, hormonal and biologic agents) was conducted according to the guidelines (16).

Patients in the CT+ cohort received anthracycline/taxane- based adjuvant CT, including epirubicin/cyclophosphamide (EC), epirubicin/cyclophosphamide followed by docetaxel (EC-T), epirubicin/cyclophosphamide followed by paclitaxel (EC-P), cyclophosphamide/methotrexate/5-fluorouracil (CMF), cyclophosphamide/epirubicin/5-fluorouracil (CEF), cyclophosphamide/epirubicin/5-fluorouracil followed by docetaxel (CEF-T), and docetaxel/cyclophosphamide (TC).

\section{Measures of clinical characteristics and outcomes}

Patient demographics and medical records were reviewed. All patients were followed up for at least six months and up to ten years. We obtained the following characteristics from FUSCC: age at diagnosis, tumor size $(\leq 2 \mathrm{~cm},>2 \mathrm{~cm})$, lymph node status (negative, positive), margin status (negative, positive), axillary surgery (sentinel node biopsy only, axillary dissection), and endocrine therapy (no, yes). We also obtained data on tumor hormone receptor and HER2 status. Table 1 displays the patient characteristics.

\section{Clinical outcomes}

The primary endpoint of this study was IBTR, which was defined as the first local recurrence in the ipsilateral (treated) breast. The date of operation was regarded as the beginning of follow-up for survival analyses. The time to an IBTR event was measured from the date of the definitive surgery until the first evidence of IBTR or the final follow-up visit if IBTR was not detected during the follow-up period. The secondary endpoint was disease-free survival (DFS), which was defined as survival from surgery to the first event, including regional recurrence and/or distant metastasis and/or non-breast cancer-specific death. Other endpoints, including local-regional failure (LRF) and overall survival (OS), were also calculated. LRF was defined as disease recurrence in the ipsilateral breast or ipsilateral regional nodes or the occurrence of a second primary tumor in the ipsilateral breast. OS was calculated from surgery to death from any cause.

\section{Statistical analyses}

Receiver operating characteristic (ROC) analyses for the prediction of 10-year IBTR and DFS were conducted to determine the optimal cutoff values of ICR and ISR in the CT+ and CT- cohorts ("survivalROC" package in $\mathrm{R})$, respectively. These values were 47 days $(\mathrm{AUC}=0.549$ ) and 69 days (AUC $=0.686$ ) in the CT+ and CT- cohorts, 
Table 1 Patient, tumor, and treatment characteristics

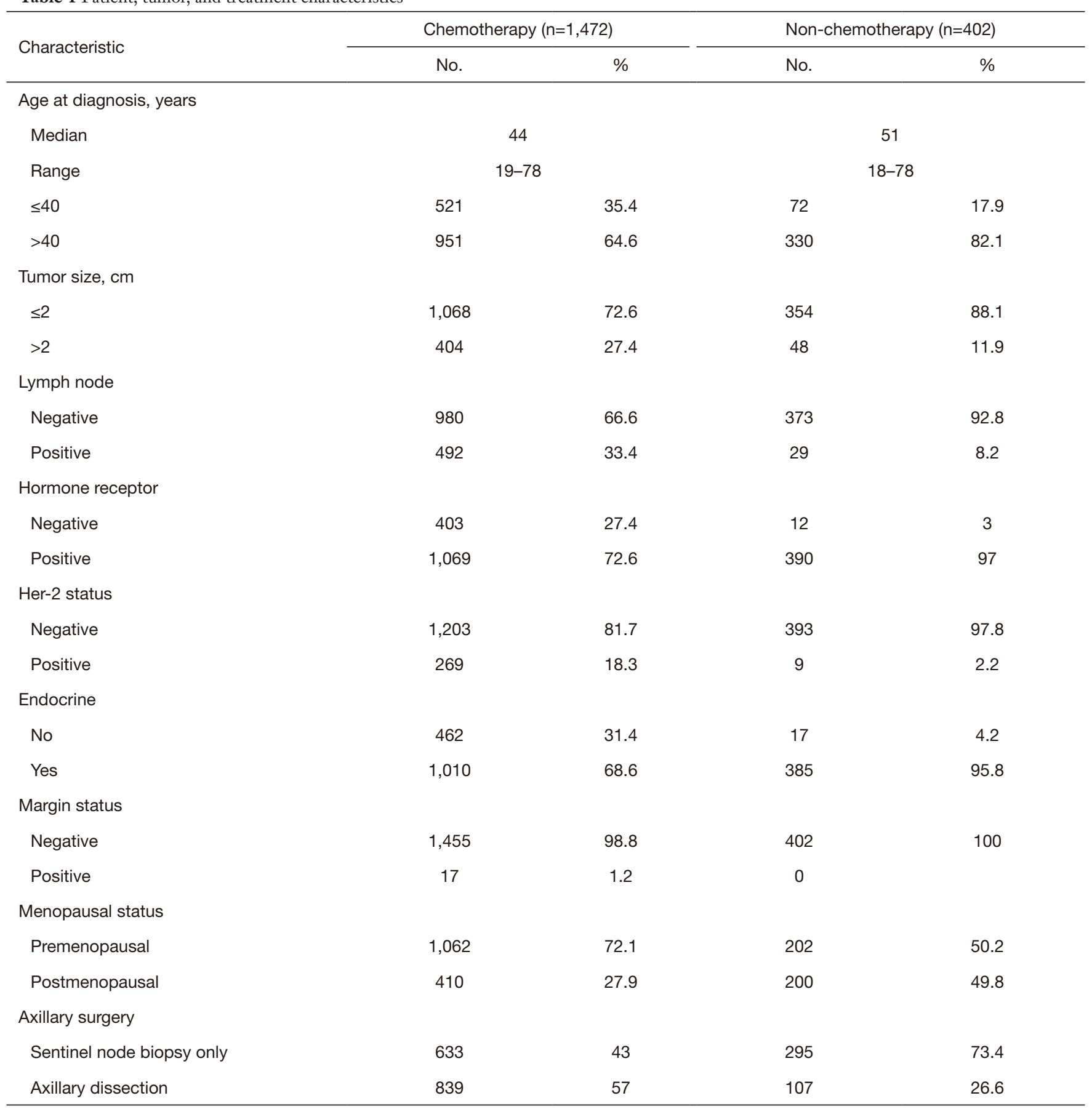

respectively (Figure S2). The patients in the CT+ and CT- cohorts were subdivided based on the cutoff values of ICR and ISR derived from ROC curves. IBTR, LRF, DFS, and OS curves were constructed using the KaplanMeier method and compared between subgroups using the log-rank test. Univariate and multivariate Cox regression analyses (forward LR selection) were performed to adjust and identify clinical factors that were used to investigate clinical outcomes. Hazard ratios (HRs) were estimated from univariate and multivariate regression analyses. A two-sided 
$\mathrm{P}<0.05$ was considered statistically significant. GraphPad Prism (version 7.0.0; GraphPad Software, San Diego, CA, USA), SPSS (version 23.0; IBM Corporation, Armonk, NY, USA), and R (version 3.6.1) were used for all analyses.

\section{Results}

\section{Patient and treatment characteristics}

Table 1 details the patient and treatment characteristics. In this cohort of patients after BCS $(n=1,874)$, the median age was 45 years (range, 18-78 years), and the median followup time was 56 months (IQR, 40-74 months). The majority of the patients $(94.1 \%)$ had stage I or stage II breast cancer. Overall, 111 patients $(5.9 \%)$ were categorized as stage III. There were $1,472(78.55 \%)$ patients in the CT+ cohort, with a median ICR of 28 days. Among 402 patients (21.45\%) in the CT-group, the median ISR was 42 days. RT was initiated within 47 days after completion of adjuvant CT in 1,296 patients (88.0\%), whereas 176 patients (12.0\%) began postoperative RT over 47 days. RT was initiated within 69 days after surgery in 352 patients $(87.5 \%)$, whereas 50 patients $(12.5 \%)$ experienced delays in RT more than 69 days after surgery. Patients with delayed initiation of postoperative RT were likely to be elderly and have positive hormone receptors, a negative HER2 status, and negative lymph nodes as well as a tumor size less than or equal to $2 \mathrm{~cm}$, both in the CT+ cohort and CT- cohort.

\section{Clinical outcomes of all patients}

Overall, there were 30 IBTR events in our final cohort (CT+ plus CT-, $n=1,874$ ), and the 5 -year cumulative incidence of IBTR was $1.4 \%$. The numbers of patients who experienced LRF, disease recurrence (regional recurrence and/or distant metastasis) and death during the follow-up period were 36 , 169 , and 43 , respectively. The 5 -year cumulative incidence rates of LRF, disease recurrence and death were $1.6 \%, 9.5 \%$, and $1.6 \%$, respectively. Of the 1,472 patients in the CT+ group, 34, 155, and 38 patients experienced LRF, disease recurrence and death, respectively. Among the 402 patients in the CT-group, 2, 14, and 5 patients experienced LRF, disease recurrence, and death, respectively.

\section{Clinical outcomes of CT+ patients}

In CT+ patients, the 5-year cumulative incidence rates of IBTR, LRF, disease recurrence and death were $1.3 \%, 1.7 \%$,
$10.6 \%$, and $1.7 \%$, respectively, in the early ICR subgroup ( $\leq 47$ days). They were $3.3 \%, 3.4 \%, 13.4 \%$, and $1.9 \%$ in the subgroup with delayed ICR ( $>47$ days) (Table S1). Figure $1 A$ shows that the IBTR curves were significantly different between the interval groups (log-rank $\mathrm{P}=0.014$ for $\leq 47 v s$. $>47$ days). Analyses of LRF, DFS, and OS outcomes were not statistically significant between the interval groups ( $\leq 47$ vs. $>47$ days) (log-rank $\mathrm{P}=0.082, \mathrm{P}=0.324, \mathrm{P}=0.411$; Figure $1 B-1 D)$. In a multivariable Cox model for IBTR in the $\mathrm{CT}+$ group (Table $\mathrm{S} 2)$, age $(\mathrm{P}=0.016)$, HER2 status $(\mathrm{P}<0.001)$, and hormone receptor status $(\mathrm{P}<0.001)$ were statistically significant. No other factor was statistically significant.

\section{Subgroup analysis of the clinical outcomes of CT+ patients}

Subsequently, the study investigated the influence of ICR ( $\leq 47$ vs. $>47$ days) on IBTR according to breast cancer subtypes. Figure $2 A$ presents the IBTR comparisons between the two interval groups in the hormone receptornegative patients who initiated postoperative RT more than 47 days after the completion of adjuvant CT; these patients had more adverse outcomes than those who began postoperative RT within 47 days in the hormone receptornegative subgroup $(\mathrm{P}=0.027)$. This was also confirmed by univariate and multivariate analyses (Table 2). The independent prognostic factors for IBTR were age (HR of 0.334; $95 \%$ CI, 0.124 to $0.903 ; \mathrm{P}=0.031)$, interval ( $\leq 47$ vs. $>47$ days) ( $\mathrm{HR}$ of $2.766 ; 95 \% \mathrm{CI}, 1.018$ to $7.518 ; \mathrm{P}=0.046$ ), and HER2 status (HR of 4.320; 95\% CI, 1.601 to 11.654; $\mathrm{P}=0.004)$. However, the cutoff point of delayed initiation of RT at 47 days was not significantly different from that of the hormone receptor-positive subgroup, as shown in Figure $2 B$ $(\mathrm{P}=0.939)$.

\section{Clinical outcomes of CT-patients}

The 5-year cumulative incidence rates of IBTR, LRF, disease recurrence, and death in the CT-group between the subgroup of early ISR ( $\leq 69$ days) and the subgroup of delayed ISR ( $>69$ days) were $0.8 \%$ vs. $2.4 \%, 0.8 \%$ vs. $0 \%$, $3.6 \%$ vs. $12.6 \%$, and $0.7 \%$ vs. $2.6 \%$, respectively (Table S1). Since only a few patients initiated RT after 69 days of surgery, no LRF was found among them. The K-M curve comparisons between interval groups ( $\leq 69 v s .>69$ days) are shown in Figure 3. Patients whose ISR exceeded 69 days had a significantly inferior rate of DFS (log-rank $\mathrm{P}=0.003$ ), and the results are presented in Figure 3C. However, clinical 
A

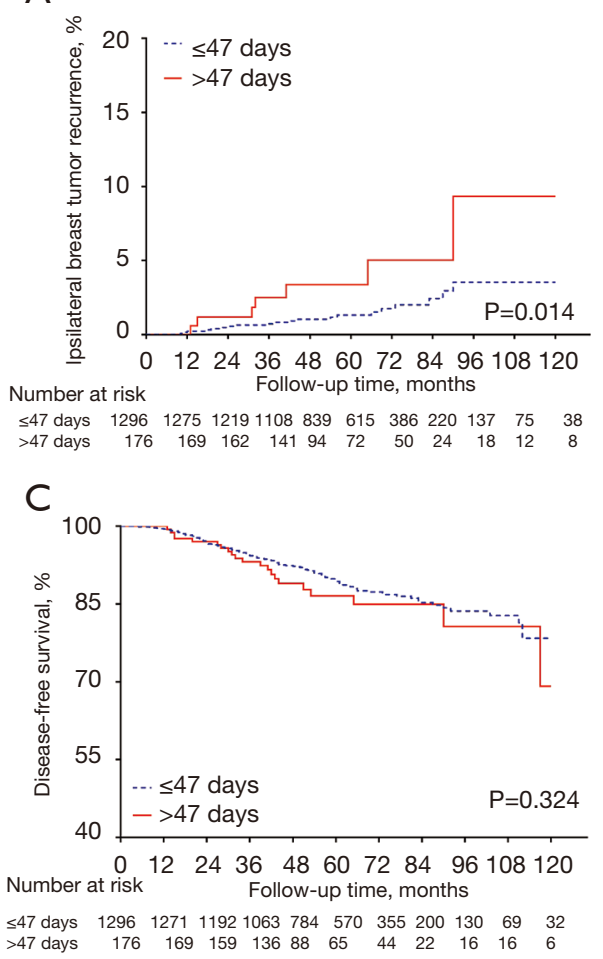

B

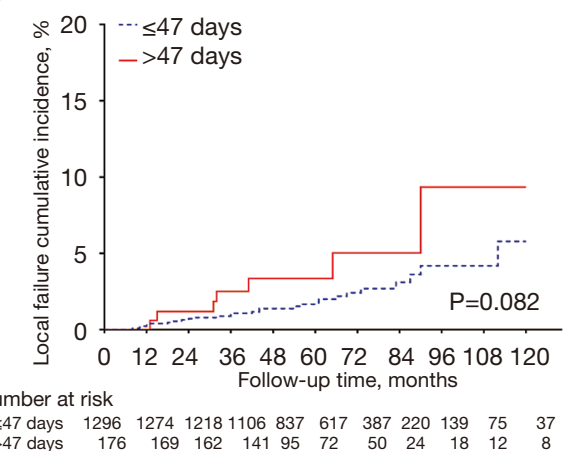

D

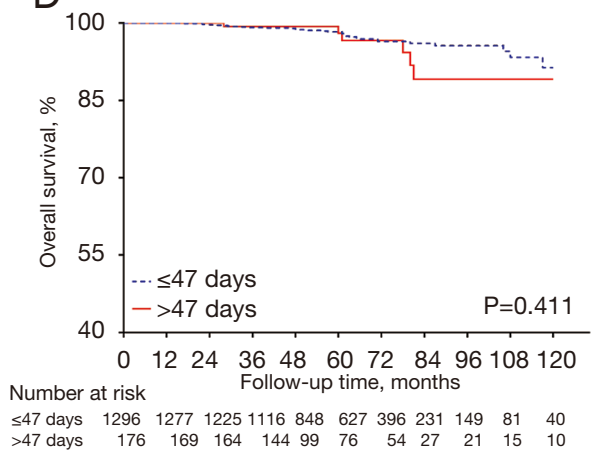

Figure $1 \mathrm{~K}-\mathrm{M}$ curves show the probability of ipsilateral breast tumor recurrence (A), local-regional failure (B), disease-free survival (C), and overall survival (D) by interval groups (days) in the $\mathrm{CT}+$ group ( $\leq 47 v s .>47$ days).

\section{A}

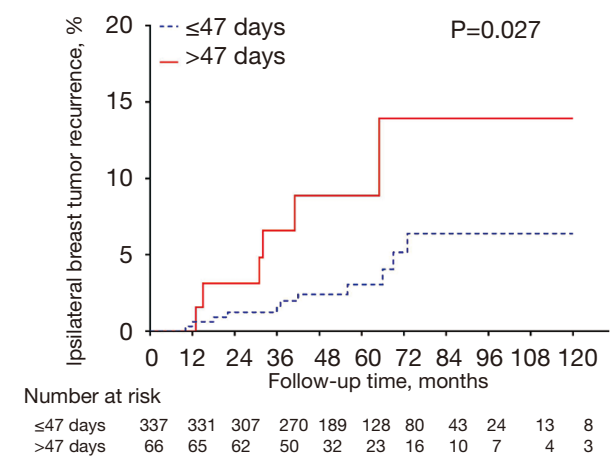

B

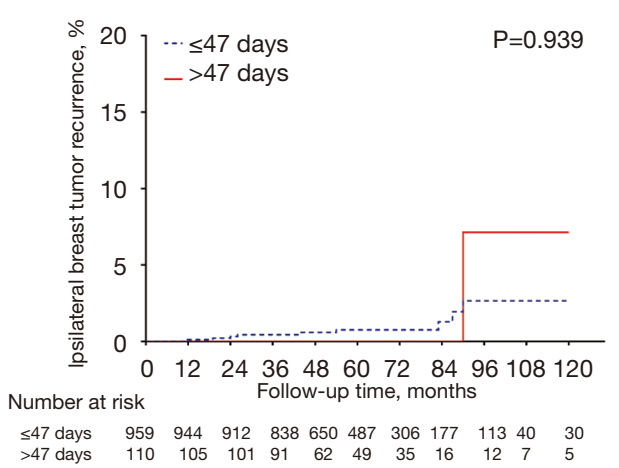

Figure 2 Kaplan-Meier plot for ipsilateral breast tumor recurrence according to the interval between completion of adjuvant chemotherapy and initiation of adjuvant radiotherapy in the hormone receptor-negative subgroup and hormone receptor-positive subgroup. Hormone receptor-negative (A); hormone receptor-positive (B).

outcomes (IBTR, LRF, and OS) did not significantly differ according to the K-M analysis of interval groups ( $\leq 69 v s$. $>69$ days) (Figure $3 A, 3 B, 3 D$ ).

In the multivariate analyses, the independent prognostic factors for DFS were age (HR of $0.234 ; 95 \%$ CI, 0.076 to
0.726; $\mathrm{P}=0.012$ ), tumor size (HR of $3.715 ; 95 \% \mathrm{CI}, 1.225$ to 11.273; $\mathrm{P}=0.020$ ), ER (HR of $0.121 ; 95 \%$ CI, 0.033 to 0.445 ; $\mathrm{P}=0.001$ ), and interval ( $\leq 69 v s .>69$ days) (HR of $6.430 ; 95 \%$ CI, 1.982 to 20.860; $\mathrm{P}=0.002$ ) (Table 3). A long delay in RT initiation of more than 69 days after surgery significantly 
Table 2 Univariate and multivariate analysis for IBTR in the hormone receptor-negative group

\begin{tabular}{|c|c|c|c|c|c|c|}
\hline Variable & \multicolumn{3}{|c|}{ Univariate } & \multicolumn{3}{|c|}{ Multivariate } \\
\hline \multicolumn{7}{|l|}{ Age (years) } \\
\hline$\leq 40$ & 1 & & & 1 & & \\
\hline$>40$ & 0.480 & $0.185-1.246$ & 0.131 & 0.334 & $0.124-0.903$ & 0.031 \\
\hline$\leq 47$ & 1 & & & 1 & & \\
\hline$>47$ & 2.935 & $1.085-7.940$ & 0.034 & 2.766 & $1.018-7.518$ & 0.046 \\
\hline \multicolumn{7}{|l|}{ Tumor size $(\mathrm{cm})$} \\
\hline$\leq 2$ & 1 & & & & & \\
\hline Negative & 1 & & & & & \\
\hline Positive & 1.290 & $0.454-3.662$ & 0.633 & - & - & 0.951 \\
\hline \multicolumn{7}{|l|}{ HER2 status } \\
\hline Negative & 1 & & & 1 & & \\
\hline Positive & 3.541 & $1.365-9.186$ & 0.009 & 4.320 & $1.601-11.654$ & 0.004 \\
\hline \multicolumn{7}{|l|}{ Margin (first section) } \\
\hline Negative & 1 & & & & & \\
\hline Positive & 0.049 & $0.000-25,601,867.29$ & 0.768 & - & - & 0.565 \\
\hline
\end{tabular}

IBTR, ipsilateral breast tumor recurrence; HR, hazard ratio; HER2, human epidermal growth factor receptor 2; Cl, confidence interval.

decreased DFS.

\section{Discussion}

Since the risks of IBTR and DFS were not cumulative, IBTR events and distant recurrence events occurred beyond the threshold value. In addition, the current study aimed to determine a reasonable timing for treatment delay. To state the results more directly, the final cutoff point for interval was selected as 47 days in the CT+ group and 69 days in the CT- group. Some studies related to delayed timing of initiation CT had cutoff points of 6 to 8 weeks after the previous treatment $(17,18)$. Moreover, a retrospective study reported that breast tumor volumes increased over a mean interval of 56.9 days, which may explain why patients who initiated RT before 47 days had better prognoses than those who initiated RT after 47 days (19).

For breast cancer in the early stage, surgery can remove the disease detected in or around the breast or regional lymph nodes, but undetected deposits of disease remain. The role of RT in optimizing local control after surgery is well established (20). However, the optimal timing of RT therapy remains controversial. Huang et al. (21) reported that the 5-year local recurrence rate (LRR) was significantly higher in patients treated with adjuvant RT for breast cancer more than 8 weeks after surgery than in those treated within 8 weeks of surgery. Since an increasing number of studies have reported that delayed RT therapy is associated with adverse outcomes in patients after BCS, the timing of $\mathrm{RT}$ is important. 
A

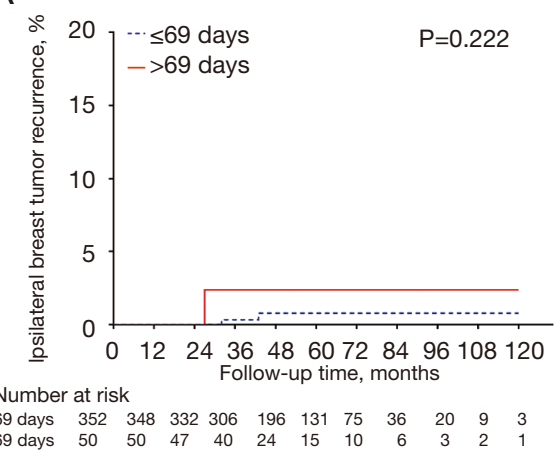

C

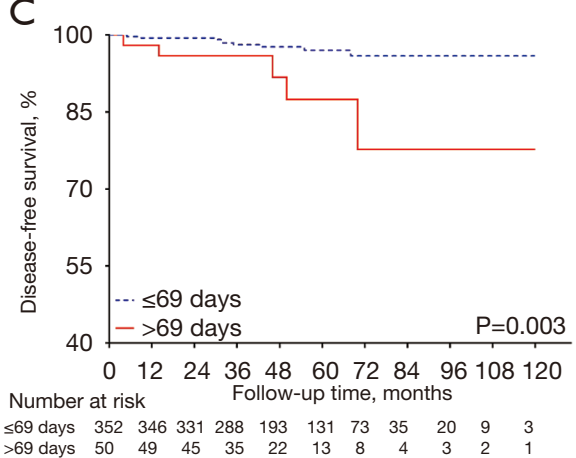

B
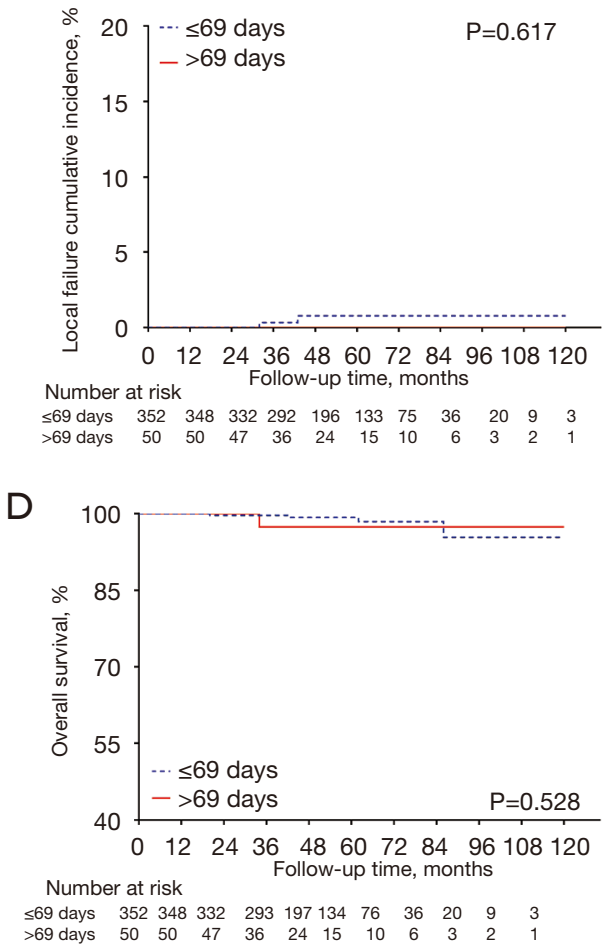

Figure 3 Kaplan-Meier plot for ipsilateral breast tumor recurrence, local-regional failure, disease-free survival, and overall survival according to the interval between surgery and initiation of adjuvant radiotherapy in the CT-group. (A) The Kaplan-Meier curves for ipsilateral breast tumor recurrence for the two groups: $\leq 69$ and $>69$ days from definitive surgery to the start of adjuvant radiotherapy; (B-D) local-regional failure, disease-free survival, and overall survival, respectively.

Our study demonstrated that patients in the CT+ group who waited longer than 47 days had increased rates of IBTR compared with patients who started postoperative RT within 47 days after adjuvant CT, especially in the hormone receptor-negative patient subgroup. In addition, our findings suggested that an ISR of more than 69 days decreased DFS compared with patients who started RT within 69 days after surgery only for patients treated with BCS plus RT.

The optimal sequence to integrate $\mathrm{CT}$ and $\mathrm{RT}$ in the adjuvant treatment of breast cancer has been investigated for decades (22-24). Given the concerns about increased distant metastases, if radiation is administered first and there are concerns related to excessive toxicity with concurrent treatment, postoperative RT will be administered after completion of adjuvant CT (16).

Many studies have suggested that delaying the initiation of adjuvant CT may decrease the benefit of cytotoxic systemic therapies and lead to adverse outcomes (25-27). Yu et al. (17) demonstrated that prolonged initiation of adjuvant CT ( $\leq 4$ vs. $>8$ weeks) significantly decreased DFS and OS, particularly among patients with triple negative and HER2 + tumors. Based on these studies, we excluded patients whose interval between surgery and the first dose of adjuvant CT exceeded 8 weeks to exclude the influence of delayed initiation of adjuvant CT in our CT+ cohort.

Delayed initiation of RT led to a poor outcome when adjuvant CT was administered first. A review by Tsoutsou et al. (28) suggested that postoperative RT should be initiated within 7 months (28 weeks) of adjuvant CT after surgery. Similar outcomes were reported by Ruo Redda $e t a l$. (7). Based on a literature review, ISR has been widely used to evaluate the influence of delayed treatment on outcomes regardless of the kind of adjuvant CT administered in patients with breast cancer. The reason that we did not use ISR to evaluate the impact of delayed RT on the clinical outcomes of patients who underwent adjuvant CT was that ISR varied with different regimens of adjuvant CT. Patients in our institution received EC, EC-T, EC-P, CMF, CEF, CEF-T, and TC, which spanned from 9 to 
Table 3 Univariate and multivariate analysis for DFS in the CT-group

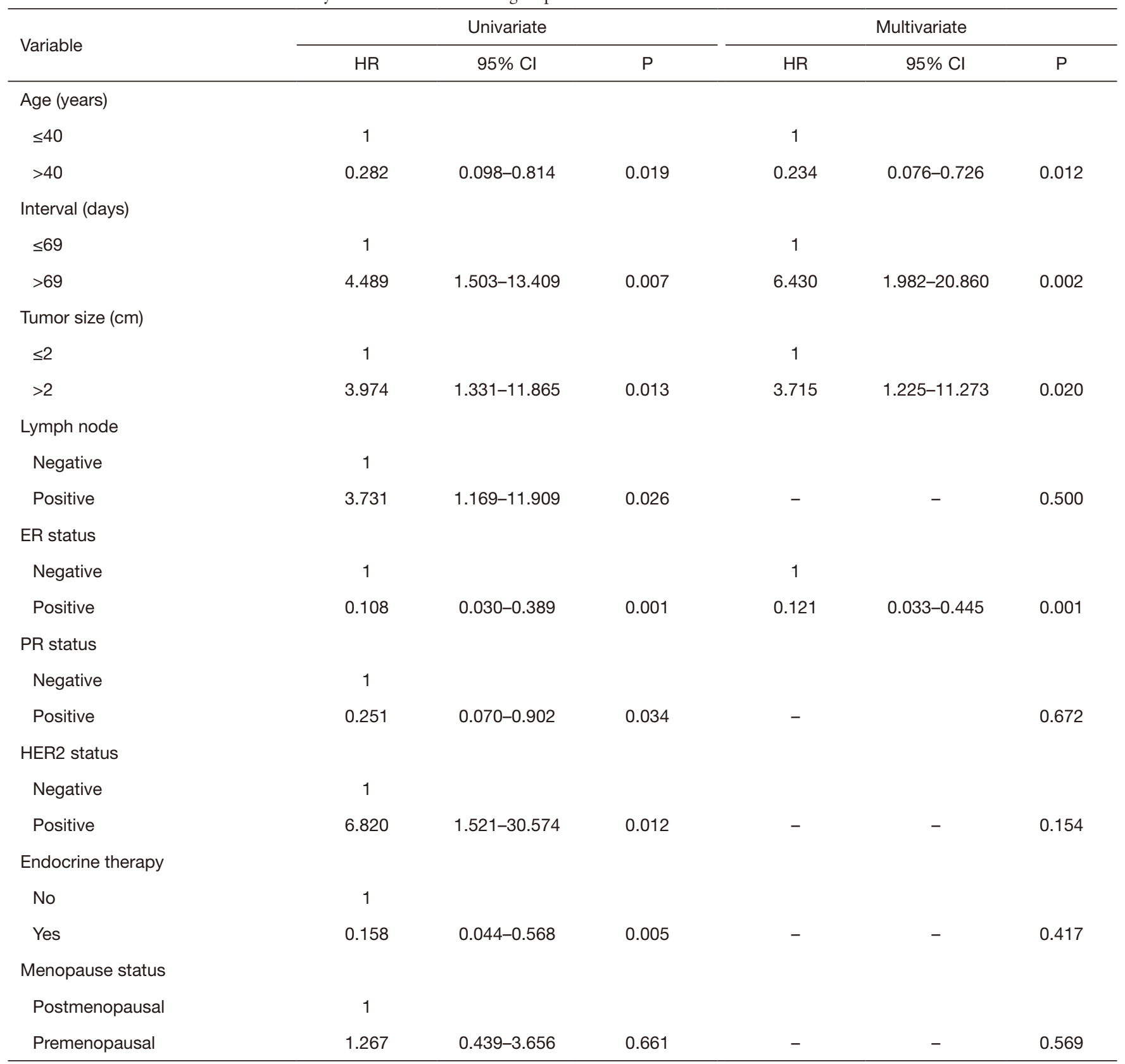

DFS, disease-free survival; ER, estrogen receptor; PR, progesterone receptor; HR, hazard ratio; HER2, human epidermal growth factor receptor 2; $\mathrm{Cl}$, confidence interval.

21 weeks depending on the risk of recurrence at diagnosis. We suggest that postoperative RT should be initiated within 47 days subsequent to the completion of CT in the current study. In other words, if patients complete adjuvant $\mathrm{CT}$ on time, we recommend that the initiation of postoperative RT should not exceed 15.5-27.5 weeks
(9-21 weeks of chemotherapy +47 days) after surgery, which is in accordance with the above-mentioned literature and the consensus that postoperative RT should be initiated within 28 weeks.

In our study, delayed initiation of RT was associated with increased IBTR, particularly in patients whose hormone 
receptor status was negative. One possible explanation was that hormone receptor-negative tumors had more aggressive tumor characteristics than hormone receptorpositive tumors (19). In addition, hormone receptor-negative tumors were more sensitive to adjuvant CT than hormone receptor-positive tumors, and the risk of IBTR in patients with hormone receptor-positive tumors may be reduced by endocrine therapy (ET), which could decrease the risk of IBTR in both early and local advanced breast cancer (29-31). Consequently, the timing of RT was quite important for patients with hormone receptor-negative breast cancer; for this reason, we recommend that these patients should initiate postoperative RT within 47 days once adjuvant CT is completed. In the present study, the independent prognostic factors for IBTR were young age, delayed interval, and positive HER2 status. Our findings are in accordance with published studies that also reported that younger patients had poorer outcomes than older patients $(32,33)$.

We attempted to correct for the effects of differences in treatment, clinical, and pathologic variables by adding hormone receptor and HER2 status into the Cox proportional hazards model. However, the inclusion of prognostic variables (especially hormone receptors) may be criticized, as they violate the proportional hazard assumption of a constant ratio over time when assessing the risk of recurrence between different values. Nevertheless, this was a common problem. The latest studies have proven that the expression of ER is indicative of a favorable prognosis $0-5$ years after diagnosis but is significantly associated with a high risk of recurrence in years 5-10 $(34,35)$. Our median follow-up was only 56 months (less than 5 years), which was not sufficient to explain the difference in the complex prognostic relevance of the estrogen receptor in the proportional hazard model.

Our study concluded that a long interval ( $>69$ days) decreased the DFS rate in the CT- cohort. In agreement with the results reported by Olivotto et al. (36), women with early-stage breast cancer who were not receiving adjuvant CT and who waited longer than 20 weeks for RT had higher rates of local and distant recurrence and adverse breast cancer-specific survival than those who started postoperative RT within 4 to 8 weeks after surgery. The majority of the patients $(97.0 \%)$ were diagnosed with hormone receptor-positive breast cancer in the CT- cohort, which is also why we did not perform subgroup analyses based on hormone status in the CT- cohort. A total of $98.7 \%$ of patients diagnosed with hormone receptor-positive tumors underwent adjuvant ET after postoperative RT. The use of adjuvant ET reduced the risk of IBTR, which may explain why the IBTR rate was unfavorable. At the same time, delayed RT may decrease the benefit of adjuvant ET. In other words, CT-patients who experience prolonged initiation of RT may experience delayed systemic therapy, which may lead to disease recurrence for those patients. This could explain why the DFS results were favorable in the CT- cohort. However, van Maaren et al. (37) reported that patients who received postoperative RT followed by CT and/or ET showed better DFS for a time interval of $>55$ days than for a time interval of $<42$ days. The reasons for the inconsistent results between the above-mentioned studies and ours are likely complex and multifactorial. First, van Maaren et al. mentioned that patients in the $<42$-day group had larger tumors and higher grades than those in the other groups. These patients with poor prognoses consequently had a shorter interval between surgery and RT, which may indicate poor DFS. Of note, ET followed by RT is recommended in our institution, but ET starts before, concurrently with, or following RT in the Netherlands. The different intervals at which patients received ET could explain the lack of consistent results between the former study and our current study. Overall, these reasons may contribute to the difference in the results between the former studies and ours.

Our study has both limitations and strengths. The strength of our study is that we selected the ICR as our main study point to balance the bias of different cycles of CT. This interval might be more suitable to investigate the optimal time to initiate RT for patients who receive both adjuvant CT and postoperative RT after BCS. This study provided new insights into the optimal ICR.

The limitations of this study included its retrospective nature, nonrandomized design and treatment selection biases. Our analyses were based on a single institution study with a relatively short median follow-up of 56 months. Further longer follow-up periods are warranted to validate long-term outcomes before such clinically available options become guidelines. In addition, because of the relatively small sample size, our multiple adjustment analysis was limited in the CT- cohort. A larger sample size dataset will be required to validate our current results.

\section{Conclusions}

In conclusion, the present study sought to organize and critically present the current data on the maximum acceptable delay of RT administration. We recommend that 
patients, especially those with hormone receptor-negative tumors, initiate RT within 47 days after they complete BCS and adjuvant CT. Patients who receive RT without adjuvant CT after BCS may initiate RT within 69 days after surgery. Based on this retrospective cohort, we recommend that patients initiate RT within the recommended interval.

\section{Acknowledgments}

The data used in this study were supported by the Fudan University Shanghai Cancer Center in China. We thank American Journal Experts (AJE) for English language editing. We thank all of the subjects who were included in this study for their participation.

Funding: This work was supported by the National Key Research and Development Program of China (2017YFC0108904) and the Fudan University Shanghai Cancer Center (YJ201809).

\section{Footnote}

Reporting Checklist: We present the following article/case in accordance with the STROBE reporting checklist. Available at https://dx.doi.org/10.21037/gs-20-717

Data Sharing Statement: Available at https://dx.doi. org/10.21037/gs-20-717

Conflicts of Interest: All authors have completed the ICMJE uniform disclosure form (available at https://dx.doi. org/10.21037/gs-20-717). The authors have no conflicts of interest to declare.

Ethical Statement: The authors are accountable for all aspects of the work in ensuring that questions related to the accuracy or integrity of any part of the work are appropriately investigated and resolved. All procedures performed in this study involving human participants followed the ethical standards of the Ethical Committee Review Board of Fudan University Shanghai Cancer Center (No. IRB050432-4-1911D). The study was conducted in accordance with the Declaration of Helsinki (as revised in 2013). Informed consent was obtained from all individual participants included in the study.

Open Access Statement: This is an Open Access article distributed in accordance with the Creative Commons Attribution-NonCommercial-NoDerivs 4.0 International
License (CC BY-NC-ND 4.0), which permits the noncommercial replication and distribution of the article with the strict proviso that no changes or edits are made and the original work is properly cited (including links to both the formal publication through the relevant DOI and the license). See: https://creativecommons.org/licenses/by-nc-nd/4.0/.

\section{References}

1. Julian TB, Venditti CA, Duggal S. Landmark clinical trials influencing surgical management of non-invasive and invasive breast cancer. Breast J 2015;21:60-6.

2. Fisher B, Anderson S, Bryant J, et al. Twenty-year followup of a randomized trial comparing total mastectomy, lumpectomy, and lumpectomy plus irradiation for the treatment of invasive breast cancer. $\mathrm{N}$ Engl J Med 2002;347:1233-41.

3. Veronesi U, Cascinelli N, Mariani L, et al. Twentyyear follow-up of a randomized study comparing breastconserving surgery with radical mastectomy for early breast cancer. N Engl J Med 2002;347:1227-32.

4. Litière $S$, Werutsky G, Fentiman IS, et al. Breast conserving therapy versus mastectomy for stage I-II breast cancer: 20 year follow-up of the EORTC 10801 phase 3 randomised trial. Lancet Oncol 2012;13:412-9.

5. Knauerhase H, Strietzel M, Gerber B, et al. Tumor location, interval between surgery and radiotherapy, and boost technique influence local control after breastconserving surgery and radiation: retrospective analysis of monoinstitutional long-term results. Int J Radiat Oncol Biol Phys 2008;72:1048-55.

6. Shurell E, Olcese C, Patil S, et al. Delay in radiotherapy is associated with an increased risk of disease recurrence in women with ductal carcinoma in situ. Cancer 2018;124:46-54.

7. Ruo Redda MG, Verna R, Guarneri A, et al. Timing of radiotherapy in breast cancer conserving treatment. Cancer Treat Rev 2002;28:5-10.

8. Punglia RS, Saito AM, Neville BA, et al. Impact of interval from breast conserving surgery to radiotherapy on local recurrence in older women with breast cancer: retrospective cohort analysis. BMJ 2010;340:c845.

9. Froud PJ, Mates D, Jackson JS, et al. Effect of time interval between breast-conserving surgery and radiation therapy on ipsilateral breast recurrence. Int J Radiat Oncol Biol Phys 2000;46:363-72.

10. Caponio R, Ciliberti MP, Graziano G, et al. Waiting time for radiation therapy after breast-conserving surgery in 
early breast cancer: a retrospective analysis of local relapse and distant metastases in 615 patients. Eur J Med Res 2016;21:32.

11. Zhang WW, Wu SG, Sun JY, et al. Long-term survival effect of the interval between mastectomy and radiotherapy in locally advanced breast cancer. Cancer Manag Res 2018;10:2047-54.

12. Corradini S, Niemoeller OM, Niyazi M, et al. Timing of radiotherapy following breast-conserving surgery: outcome of 1393 patients at a single institution. Strahlenther Onkol 2014;190:352-7.

13. Koh HK, Shin KH, Kim K, et al. Effect of Time Interval between Breast-Conserving Surgery and Radiation Therapy on Outcomes of Node-Positive Breast Cancer Patients Treated with Adjuvant Doxorubicin/ Cyclophosphamide Followed by Taxane. Cancer Res Treat 2016;48:483-90.

14. Harvey JM, Clark GM, Osborne CK, et al. Estrogen receptor status by immunohistochemistry is superior to the ligand-binding assay for predicting response to adjuvant endocrine therapy in breast cancer. J Clin Oncol 1999;17:1474-81.

15. Wolff AC, Hammond ME, Schwartz JN, et al. American Society of Clinical Oncology/College of American Pathologists guideline recommendations for human epidermal growth factor receptor 2 testing in breast cancer. J Clin Oncol 2007;25:118-45.

16. Atag Akyurek E. National Comprehensive Cancer Network (NCCN) version 2 2019. 2019.

17. Yu KD, Fan L, Qiu LX, et al. Influence of delayed initiation of adjuvant chemotherapy on breast cancer survival is subtype-dependent. Oncotarget 2017;8:46549-56.

18. He X, Ye F, Zhao B, et al. Risk factors for delay of adjuvant chemotherapy in non-metastatic breast cancer patients: A systematic review and meta-analysis involving 186982 patients. PLoS One 2017;12:e0173862.

19. Nakashima K, Uematsu T, Takahashi K, et al. Does breast cancer growth rate really depend on tumor subtype? Measurement of tumor doubling time using serial ultrasonography between diagnosis and surgery. Breast Cancer 2019;26:206-14.

20. Clarke M, Collins R, Darby S, et al. Effects of radiotherapy and of differences in the extent of surgery for early breast cancer on local recurrence and 15-year survival: an overview of the randomised trials. Lancet 2005;366:2087-106.

21. Huang J, Barbera L, Brouwers M, et al. Does delay in starting treatment affect the outcomes of radiotherapy? A systematic review. J Clin Oncol 2003;21:555-63.

22. Buchholz TA, Austin-Seymour MM, Moe RE, et al. Effect of delay in radiation in the combined modality treatment of breast cancer. Int J Radiat Oncol Biol Phys 1993;26:23-35.

23. Bellon JR, Come SE, Gelman RS, et al. Sequencing of chemotherapy and radiation therapy in early-stage breast cancer: updated results of a prospective randomized trial. J Clin Oncol 2005;23:1934-40.

24. Toledano A, Azria D, Garaud P, et al. Phase III trial of concurrent or sequential adjuvant chemoradiotherapy after conservative surgery for early-stage breast cancer: final results of the ARCOSEIN trial. J Clin Oncol 2007;25:405-10.

25. Li S, Ma D, Shi HH, et al. The effect of delayed adjuvant chemotherapy on relapse of triple-negative breast cancer. J Thorac Dis 2018;10:2837-41.

26. Nurgalieva ZZ, Franzini L, Morgan RO, et al. Impact of timing of adjuvant chemotherapy initiation and completion after surgery on racial disparities in survival among women with breast cancer. Med Oncol 2013;30:419.

27. Chavez-MacGregor M, Clarke CA, Lichtensztajn DY, et al. Delayed Initiation of Adjuvant Chemotherapy Among Patients With Breast Cancer. JAMA Oncol 2016;2:322-9.

28. Tsoutsou PG, Koukourakis MI, Azria D, et al. Optimal timing for adjuvant radiation therapy in breast cancer: a comprehensive review and perspectives. Crit Rev Oncol Hematol 2009;71:102-16.

29. Wapnir IL, Dignam JJ, Fisher B, et al. Longterm outcomes of invasive ipsilateral breast tumor recurrences after lumpectomy in NSABP B-17 and B-24 randomized clinical trials for DCIS. J Natl Cancer Inst 2011;103:478-88.

30. Allred DC, Anderson SJ, Paik S, et al. Adjuvant tamoxifen reduces subsequent breast cancer in women with estrogen receptor-positive ductal carcinoma in situ: a study based on NSABP protocol B-24. J Clin Oncol 2012;30:1268-73.

31. Fisher B, Jeong JH, Bryant J, et al. Treatment of lymphnode-negative, oestrogen-receptor-positive breast cancer: long-term findings from National Surgical Adjuvant Breast and Bowel Project randomised clinical trials. Lancet 2004;364:858-68.

32. Ozkaya Akagunduz O, Ergen A, Erpolat P, et al. Local recurrence outcomes after breast conserving surgery and adjuvant radiotherapy in ductal carcinoma in situ of the breast and a comparison with ECOG E5194 study. Breast 2018;42:10-4. 
33. Vujovic O, Cherian A, Yu E, et al. The effect of timing of radiotherapy after breast-conserving surgery in patients with positive or close resection margins, young age, and node-negative disease, with long term follow-up. Int J Radiat Oncol Biol Phys 2006;66:687-90.

34. Richman J, Dowsett M. Beyond 5 years: enduring risk of recurrence in oestrogen receptor-positive breast cancer. Nat Rev Clin Oncol 2019;16:296-311.

35. Dowsett M, Sestak I, Buus R, et al. Estrogen Receptor Expression in 21-Gene Recurrence Score Predicts Increased Late Recurrence for Estrogen-Positive/HER2-Negative

Cite this article as: Ma X, Chen J, Ma D, Jiang Y, Liu Z, Chen S, Zhang Y, Shen Y, Yu X, Yang Z, Li S, Mo M, Qian Y, Liu G, Wu J, Shao Z, Yu K, Di G. Delayed initiation of radiation therapy is associated with inferior outcomes for breast cancer patients with hormone receptor-negative tumors after breast-conserving surgery. Gland Surg 2021;10(9):2631-2643. doi: 10.21037 /gs-20-717
Breast Cancer. Clin Cancer Res 2015;21:2763-70.

36. Olivotto IA, Lesperance ML, Truong PT, et al. Intervals longer than 20 weeks from breast-conserving surgery to radiation therapy are associated with inferior outcome for women with early-stage breast cancer who are not receiving chemotherapy. J Clin Oncol 2009;27:16-23.

37. van Maaren MC, Bretveld RW, Jobsen JJ, et al. The influence of timing of radiation therapy following breastconserving surgery on 10-year disease-free survival. Br J Cancer 2017;117:179-88. 


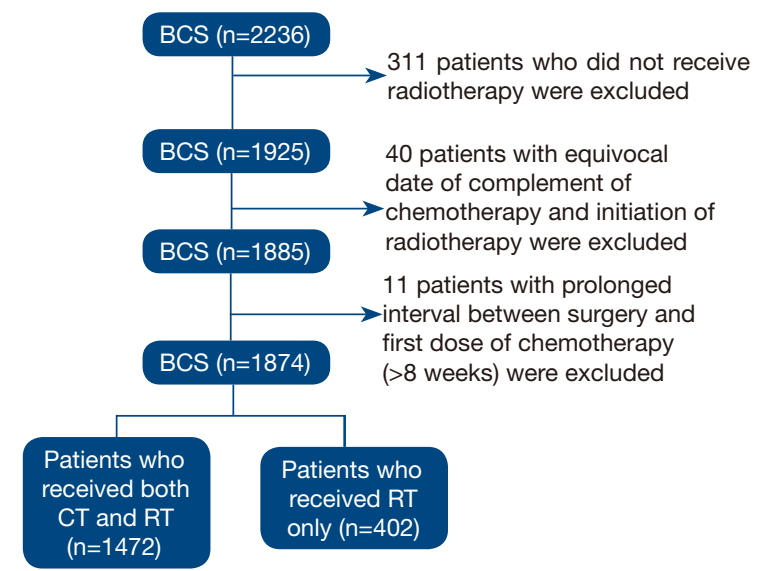

Figure S1 Enrolled and excluded study patients.
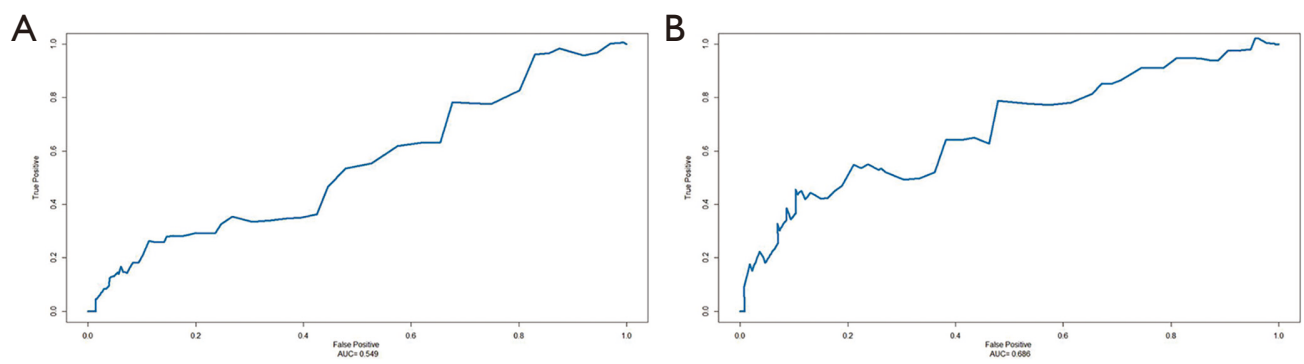

Figure S2 Area under the curve (AUC) of the survival ROC. AUC of the 10-year survival ROC curve for IBTR in CT+ cohorts (A), AUC of the 10-year survival ROC curve for DFS in CT- cohorts (B).

Table S1 Crude and actuarial results in the CT+ and CT-group

\begin{tabular}{|c|c|c|}
\hline Clinical outcomes & Chemotherapy $(n=1472)(\%)$ & Non-chemotherapy $(n=402)(\%)$ \\
\hline \multicolumn{3}{|l|}{ Ipsilateral breast tumor recurrence } \\
\hline 5-year crude results & 1.3 & 0.7 \\
\hline \multirow[t]{2}{*}{ 5-year cumulative incidence of IBTR } & 1.3 ( $\leq 47$ days $)$ & 0.8 ( $\leq 69$ days $)$ \\
\hline & 3.3 (>47 days) & 2.4 (>69 days) \\
\hline \multicolumn{3}{|l|}{ Local-regional failure } \\
\hline 5 -year crude results & 1.6 & 0.5 \\
\hline \multirow[t]{2}{*}{ 5-year cumulative incidence of LRF } & 1.7 ( $\leq 47$ days) & 0.8 ( $\leq 69$ days) \\
\hline & 3.4 (>47 days) & 0 (>69 days) \\
\hline \multicolumn{3}{|l|}{ Disease-free survival } \\
\hline 5-year crude results & 8.7 & 3 \\
\hline \multirow[t]{2}{*}{ 5-year cumulative incidence of disease recurrence } & 10.6 ( $\leq 47$ days $)$ & 3.0 ( $\leq 69$ days $)$ \\
\hline & 13.4 (>47 days) & 12.6 (>69 days) \\
\hline \multicolumn{3}{|l|}{ Overall survival } \\
\hline 5-year crude results & 1.3 & 0.7 \\
\hline \multirow[t]{2}{*}{ 5-year cumulative incidence of death } & 1.7 ( $\leq 47$ days $)$ & 0.7 ( $\leq 69$ days) \\
\hline & 1.9 (>47 days) & 2.6 (>69 days) \\
\hline
\end{tabular}

IBTR, ipsilateral breast tumor recurrence; LRF, local-regional failure. 
Table S2 Univariate and multivariate analysis for IBTR in the CT+ group

\begin{tabular}{|c|c|c|c|c|c|c|}
\hline \multirow{2}{*}{ Variable } & \multicolumn{3}{|c|}{ Univariate } & \multicolumn{3}{|c|}{ Multivariate } \\
\hline & HR & $95 \% \mathrm{Cl}$ & $\mathrm{P}$ & $\mathrm{HR}$ & $95 \% \mathrm{Cl}$ & $\mathrm{P}$ \\
\hline \multicolumn{7}{|l|}{ Age } \\
\hline$\leq 40$ & 1 & & & 1 & & \\
\hline$>40$ & 0.504 & $0.237-1.071$ & 0.075 & 0.387 & $0.179-0.836$ & 0.016 \\
\hline \multicolumn{7}{|l|}{ Interval (days) } \\
\hline$\leq 47$ & 1 & & & & & \\
\hline$>47$ & 2.795 & $1.181-6.611$ & 0.019 & - & - & 0.076 \\
\hline \multicolumn{7}{|l|}{ Tumor size (cm) } \\
\hline$\leq 2$ & 1 & & & & & \\
\hline$>2$ & 1.590 & $0.728-3.473$ & 0.245 & - & - & 0.326 \\
\hline \multicolumn{7}{|l|}{ Lymph node } \\
\hline Negative & 1 & & & & & \\
\hline Positive & 0.773 & $0.338-1.766$ & 0.773 & - & - & 0.743 \\
\hline \multicolumn{7}{|l|}{ Her2 status } \\
\hline Negative & 1 & & & 1 & & \\
\hline Positive & 5.478 & $2.565-11.697$ & 0.000 & 4.984 & $2.297-10.814$ & 0.000 \\
\hline \multicolumn{7}{|l|}{ Margin (first section) } \\
\hline Negative & 1 & & & & & \\
\hline Positive & 0.049 & $0.000-236,174.251$ & 0.701 & - & - & 0.473 \\
\hline \multicolumn{7}{|l|}{ Endocrine therapy } \\
\hline No & 1 & & & & & \\
\hline Yes & 0.201 & $0.090-0.448$ & 0.000 & - & - & 0.465 \\
\hline \multicolumn{7}{|l|}{ Menopause status } \\
\hline Postmenopausal & 1 & & & & & \\
\hline Premenopausal & 1.689 & $0.639-4.460$ & 0.290 & - & - & 0.340 \\
\hline \multicolumn{7}{|l|}{ Hormone receptor } \\
\hline Negative & 1 & & & 1 & & \\
\hline Positive & 0.190 & $0.087-0.415$ & 0.000 & 0.221 & $0.100-0.487$ & 0.000 \\
\hline
\end{tabular}

IBTR, ipsilateral breast tumor recurrence; HR, hazard ratio; HER2, human epidermal growth factor receptor 2; $\mathrm{Cl}$, confidence interval. 